\title{
PHENOTYPIC CHARACTERIZATION AND ANTIBIOTIC SUSCEPTIBILITIES OF EWINGELLA AMERICANA AND KLUYVERA INTERMEDIA ISOLATED FROM SOAKED HIDES AND SKINS
}

\author{
EDA YAZICI ${ }^{1 *}$, MERAL BIRBIR ${ }^{2}$, PINAR CAGLAYAN ${ }^{2}$ \\ ${ }^{1}$ Marmara University, Institute of Pure and Applied Sciences, Istanbul, Turkey, *Corresponding \\ Author:eda.yazici@marun.edu.tr \\ ${ }^{2}$ Marmara University, Faculty of Arts and Sciences, Biology Department, Istanbul, Turkey,
}

\begin{abstract}
Soaked hides and skins may contain different species of family Enterobacteriaceae, originating from animal's feces, soil, and water. Some species of this family may be pathogenic to humans and animals. Hence, phenotypic characteristics and antibiotic susceptibilities of Ewingella americana and Kluyvera intermedia belonging to Enterobacteriaceae were explained in this study. While Ewingella americana was isolated from only one soaked hide, Kluyvera intermedia was isolated from both soaked hide and skin. Phenotypic characterization of these isolates was performed using API 20E test kit. Antibiotic susceptibilities of these isolates were examined by Kirby Bauer Disc Diffusion Test using piperacillin/tazobactam $(110 \mu \mathrm{g})$, amoxicillin/clavulanate $(30 \mu \mathrm{g})$, ampicillin/sulbactam $(20 \mu \mathrm{g})$, amikacin $(30 \mu \mathrm{g})$, tobramycin $(10 \mu \mathrm{g})$, kanamycin $(30 \mu \mathrm{g})$, gentamicin $(10 \mu \mathrm{g})$, streptomycin $(10 \mu \mathrm{g})$, ampicillin $(10 \mu \mathrm{g})$, imipenem $(10 \mu \mathrm{g})$, meropenem $(10 \mu \mathrm{g})$, cefoxitin $(30 \mu \mathrm{g})$, cefuroxime sodium $(30 \mu \mathrm{g})$, ceftazidime $(30 \mu \mathrm{g})$, cephalothin $(30 \mu \mathrm{g})$, ceftriaxone $(30 \mu \mathrm{g})$, norfloxacin $(10 \mu \mathrm{g})$, nalidixic acid $(30 \mu \mathrm{g})$, ofloxacin $(5 \mu \mathrm{g})$, ciprofloxacin $(10 \mu \mathrm{g})$, sulfamethoxazole-trimethoprim $(25 \mu \mathrm{g})$, aztreonam $(30 \mu \mathrm{g})$, chloramphenicol $(30 \mu \mathrm{g})$, tetracyline $(30 \mu \mathrm{g})$. Ewingella americana was resistant against aztreonam, ceftazidime, ceftriaxone but this isolate was intermediate susceptible against cefuroxime sodium, ampicillin, nalidixic acid, tetracyline and chloramphenicol. Kluyvera intermedia was found to be resistant against streptomycin, cephalothin, aztreonam, and ampicillin but it was intermediate susceptible to amikacin, kanamycin, chloramphenicol, imipenem, ceftazidime, ceftriaxone, amoxicillin/clavulanate and ciprofloxacin. Moreover, both isolates were found to be susceptible to other antibiotics. Therefore, effective antibacterial applications should be applied to kill these antibiotic resistant bacteria.
\end{abstract}

Keywords: Ewingella americana, Kluyvera intermedia, antibiotics, soaked hides and skins

\section{INTRODUCTION}

Freshly slaughtered hides/skins may have different species of microorganisms which are related to normal bacterial flora of animal's skin and feces, soil, water and air (Birbir and Ilgaz, 1996; Aslan and Birbir, 2012; Birbir et al., 2016). To prevent bacterial growth and decomposition of hides/skins, moisture contents of these organic materials are reduced by drying, salt or brine curing until the beamhouse processes(Bailey, 2003). However, if the curing process has not performed adequately, salt tolerant bacteria, halophilic microorganisms and bacterial members of family Enterobacteriaceae may grow and damage to cured and soaked hides/skins (Bailey and Birbir, 1993; Bailey and Birbir, 1996; Birbir and Ilgaz, 1996; Berber and Birbir, 2010; Aslan and Birbir, 2012; Ulusoy and Birbir, 2015; Akpolat et al., 2015; Caglayan et al., 2017).

In soaking, the hides/skins are rehydrated and dirt, salt, blood, urine, manure, and interfibrillary material have been removed from the hides/skins. Long process duration, high organic content of soaking solution support growth and bacterial activities. Hence, antimicrobial agent is added into soaking solution but high organic content of soak liquor may adversely affect the efficiency of antimicrobial agent. In the study of Rangarajan et al. (2003), species of Bacillus, Chromobacter, Pseudomonas, Clostridium, Lactobacillus and Serratia were isolated from soak liquor. It was reported that bacterial populations in first soaking process decreased rapidly but tended to increase over time. 
In another study, Pfleiderer et al. (1988) isolated bacterial species belonging to genera Proteus, Pseudomonas, Bacillus, Corynebacterium, Clostridium, Chromobacter, Lactobacillus, Micrococcus, Sarcina, Staphylococcus and Serratia from soak liquors. Moreover, bacterial species of genera Citrobacter, Edwardsiella, Enterobacter, Escherichia, Hafnia, Klebsiella, Proteus, Salmonella, Serratia and Yersinia belonging to family Enterobacteriaceae were isolated from ten salted cattle hides (Aslan and Birbir, 2012). Ulusoy and Birbir (2015) stated that total numbers of Enterobacteriaceae; total numbers of proteolytic Enterobacteriaceae; total numbers of lipolytic Enterobacteriaceae on salted hides and skins were found as between $1.7 \times 10^{4} \mathrm{cfu} / \mathrm{g}$ $4.5 \times 10^{5} \mathrm{cfu} / \mathrm{g}$ and between $1.7 \times 10^{5} \mathrm{cfu} / \mathrm{g}-1.5 \times 10^{6} \mathrm{cfu} / \mathrm{g}$; between $9.1 \times 10^{3} \mathrm{cfu} / \mathrm{g}-3.9 \times 10^{5}$ $\mathrm{cfu} / \mathrm{g}$ and between $1.2 \times 10^{5} \mathrm{cfu} / \mathrm{g}-1.1 \times 10^{6} \mathrm{cfu} / \mathrm{g}$; between $6.0 \times 10^{2} \mathrm{cfu} / \mathrm{g}-3.7 \times 10^{5} \mathrm{cfu} / \mathrm{g}$ and between $1.7 \times 10^{4} \mathrm{cfu} / \mathrm{g}-5.0 \times 10^{5} \mathrm{cfu} / \mathrm{g}$, respectively.

The family Enterobacteriaceae contains 56 genera (Arsenophonus, Biostraticola, Brenneria, Buchnera, Budvicia, Buttiauxella, Dickeya, Calymmatobacterium, Cedecea, Citrobacter, Cosenzaea, Cronobacter, Sodalis, Pragia, Edwardsiella, Enterobacillus, Enterobacter, Erwinia, Escherichia, Ewingella, Franconibacter, Gibbsiella, Hafnia, Klebsiella, Kluyvera, Leclercia, Leminorella, Levinea, Lonsdalea, Mangrovibacter, Moellerella, Morganella, Obesumbacterium, Serratia, Pantoea, Pectobacterium, Phaseolibacter, Photorhabdus, Plesiomonas, Proteus, Providencia, Raoultella, Rouxiella, Saccharobacter, Salmonella, Samsonia, Shigella, Shimwellia, Tatumella, Thorsellia, Rahnella, Trabulsiella, Wigglesworthia, Xenorhabdus, Yersinia, Yokenella) 454 named species (http://www.bacterio.net/-classifphyla.html\#bacteria). All members of the family Enterobacteriaceae are facultative anaerobic, chemoorganotrophic, nonhalophilic, non-sporulating, rod-shaped Gram-negative bacteria. Although most of the enteric bacteria may be motile by peritrichous flagella, a few of them are nonmotile. Enterobacteriaceae may be found in water, soil, animals and plants. Some species may be pathogenic to humans, animals, plants and insects. Most are oxidase negative, catalase positive and reduce nitrate to nitrite. Acid and gas are produced during fermentation of D-glucose, other carbohydrates and polyhydroxyl alcohols (Brenner and Farmer, 2005).

Newton et al. (1977) isolated Enterobacter liquefaciens, Enterobacter aerogenes, Enterobacter cloacae, Klebsiella pneumoniae, Citrobacter spp. and Serratia spp. from 85 hides. Oppong et al. (2006) isolated Citrobacter freundii and Proteus vulgaris from both raw and soaked hides. In the study of Shede et al. (2008), Escherichia coli, Proteus mirabilis, Shigella boydii, Photorhabdus luminescens, Pantoea agglomerans were isolated from raw buffalo hides.

Detection of Ewingella in vacuum-packaged meat, vegetables, molluscs, shiitake, button and oyster mushrooms, and some clinical specimens taken from blood, wounds, respiratory tracts was reported by the investigators (Muller et al.,1995; Hamilton-Miller and Shah, 2001; Reyes et al., 2004). Ewingella americana uncommonly causes infection in humans, is associated with nosocomial infections (Grimont et al., 1983; Farmer et al., 1985; Heizmann and Michel, 1991). Kluyvera intermedia, which is a potential pathogen, was isolated from potable water, surface water, soil, human samples such as stool, bile and gall blader (Pavan et al., 2005). This organism may cause blood infections, urinary tract and soft tissue infections and septic shock (Janda, 2006).

Antimicrobial agents are commonly used for human and animal health and welfare. Antimicrobial resistance influenced by widespread and inappropriate usage of antimicrobial agents in medicine, veterinary medicine and agriculture is a global public 
and animal health concern (Madigan et al., 2015; World Organization for Animal Health, 2015). Antibiotic resistance profiles of some members of Enterobacteriaceae isolated from salted hide and skin samples were examined in our previous study. In that study $70 \%$ of the hide and $68 \%$ of the sheep skin strains were found to be resistant to three or more of 24 antimicrobial agents. Resistance of the isolates to ampicillin (45\%), cefoxitin $(20 \%)$, kanamycin $(9 \%)$, ceftriaxone $(45 \%)$, meropenem $(2 \%)$, aztreonam (71\%), ceftazidime $(33 \%)$, imipenem $(4 \%)$, tetracycline $(16 \%)$, amikacin $(5 \%)$, ciprofloxacin $(5 \%)$, cephalothin $(16 \%)$, gentamicin $(5 \%)$, amoxycillin-clavulanate $(25 \%)$, tobramycin $(13 \%)$, ampicillin-sulbactam (29\%), piperacillin-tazobactam $(38 \%)$, ofloxacin (2\%), cefuroxime sodium (45\%), chloramphenicol (35\%),streptomycin $(9 \%)$, sulfamethoxazole/trimethoprim (25\%) and nalidixic-acid(42\%) was detected (Ulusoy, 2014; Birbir et al., 2016).

Although there are several studies on members of the family Enterobacteriaceae, antibiotic susceptibilities of Ewingella americana and Kluyvera intermedia isolated from soaked cattle hides and sheep skin samples treated with antimicrobial agent have not been examined yet. Hence, the goal of the study was to present phenotypic characteristics and antibiotic susceptibilities of Ewingella americana and Kluyvera intermedia.

\section{MATERIAL AND METHOD}

\section{Test Isolates}

The isolates were obtained from the soaked cattle hides and sheep skin samples using Eosin Methylene Blue Agar. Phenotypic characterization of these isolates were performed according to catalase and oxidase activities, reduction of nitrate to nitrite and API 20E test kit containing biochemical tests such as $\beta$-galactosidase, arginine dihydrolase, lysine decarboxylase, ornithine decarboxylase, utilization of citrate, production of $\mathrm{H}_{2} \mathrm{~S}$, urease, tryptophan deaminase, production of indole from tryptophan, Voges-Proskauer, production of gelatinase, fermentation of glucose, mannose, inositol, sorbitol, rhamnose, sucrose, melibiose, amygdalin, arabinose (Yazici and Birbir, 2018).

\section{Antibiotic Susceptibility Test}

One isolate of Ewingella americana and two isolates of Kluyvera intermedia were grown on Mueller Hinton Agar at $37^{\circ} \mathrm{C}$ for 24 hours and the isolates were separately inoculated into Mueller Hinton Broth and incubated at $37^{\circ} \mathrm{C}$ for 24 hours. Then, the bacterial density of these isolates was adjusted to approximately $1 \times 10^{8} \mathrm{cfu} / \mathrm{mL}$. Disc diffusion susceptibility method was used to detect antibiotic susceptibilities of the isolates (CLSI, 2014; EUCAST, 2014). 24 antimicrobial agents belonging to 9 categories such as tetracyclines (tetracyline), carbapenems (imipenem, meropenem), aminoglycosides (amikacin, kanamycin, tobramycin, streptomycin, gentamicin), monobactams (aztreonam), penicillins ( $\beta$-lactam/ $\beta$-lactamase inhibitor combinations) (piperacillin/tazobactam, amoxicillin/clavulanate, ampicillin/sulbactam, ampicillin), cephalosporins I, II and III (cefoxitin, cefuroxime sodium, ceftazidime, cephalothin, ceftriaxone), quinolones and fluoroquinolones (norfloxacin, ofloxacin, nalidixic acid, ciprofloxacin), amphenicols (chloramphenicol) and sulfonamides, dihydrofolate reductase inhibitors combinations (sulfamethoxazole/trimethoprim), were utilized in the present study. The antimicrobial agents were placed on the Mueller Hinton Agar plate 
inoculated with test isolate. After incubation at $37^{\circ} \mathrm{C}$ for 24 hours, the zones of growth inhibition around each of the test antibiotics were measured and the results were evaluated according to the criteria explained by the of Clinical and Laboratory Standards Institute (CLSI, 2014) and European Committee on Antimicrobial Susceptibiliy Testing (EUCAST, 2014). The test antimicrobial agents were purchased from Oxoid (Basingstoke, Hants, UK).

\section{RESULTS AND DISCUSSION}

While Ewingella americana was isolated from only soaked hide (CH10), Kluyvera intermedia was isolated from both soaked hide and skin samples (SS3 and CH1). Although Ewingella americana showed positive reactions of $\beta$-galactosidase, VogesProskauer, citrate utilization, catalase, nitrate reduction to nitrite, acid production from glucose and mannitol, negative reaction was detected in $\mathrm{H}_{2} \mathrm{~S}$ production, oxidase, urease, gelatinase, tryptophan deaminase, indol production, ornithine decarboxylase, lysine decarboxylase, arginine dihydrolase, acid production from inositol, sorbitol, rhamnose, sucrose, melibiose, amygdalin and arabinose (Yazici and Birbir, 2018). These biochemical test results were similar to the results mentioned in Janda (2006).

Ewingella americana was resistant to aztreonam, ceftazidime and ceftriaxone. While Ewingella americana was intermediate susceptible to cefuroxime sodium, ampicillin, nalidixic acid, tetracyline and chloramphenicol, this isolate was susceptible to tobramycin, gentamicin, kanamycin, streptomycin, amikacin, meropenem, imipenem, cephalothin, cefoxitin, ampicillin/sulbactam, amoxicillin/clavulanate, ciprofloxacin, piperacillin-tazobactam, sulfamethoxazole-trimethoprim, norfloxacin and ofloxacin in the present study. Bukhari and colleagues (2008) mentioned that E. americana, which was isolated from a patient, was resistant to ceftazidime and ceftriaxone. Our antibiotic results were similar to that study (Bukhari et al., 2008). Resistance of this isolate to ceftriaxone and aztreonam was stated by Pound et al. (2007). Moreover, Pound et al. (2007) detected that E. americana, isolated from a patient with a chronic pulmonary disease, was also resistant to amikacin, gentamicin, tobramycin, ampicillin, ampicillin/sulbactam, cefoxitin, ciprofloxacin, imipenem, and tetracycline. While the isolate was found to be intermediate susceptible to piperacillin/tazobactam, ceftazidime, susceptible to sulfamethoxazole/trimethoprim (Pound et al., 2007). In our study, the susceptibility of E. americana against aminoglycosides, carbapenems, fluoroquinolones, sulfamethoxazole/trimethoprim and cefoxitin were consistent with antibiotic test results of Stock et al. (2013). The researchers emphasized that E. americana has natural susceptibility to most antibiotics including aminoglycosides, aztreonam, cefepime, cefotaxime, trimethoprim/sulfamethoxazole, carbapenems, cefoxitin, fluoroquinolones, tetracyclines and chloramphenicol. In another study, all hide isolates of Ewingella americana were susceptible to tobramycin, gentamicin, kanamycin, streptomycin, amikacin, cefoxitin, imipenem, meropenem, piperacillin-tazobactam, ofloxacin, amoxicillin/clavulanate, norfloxacin, ciprofloxacin, cephalothin, ampicillin/sulbactam and sulfamethoxazole-trimethoprim, these isolates were detected as resistant to aztreonam, ceftazidime and ceftriaxone. Ewingella americana was found to be intermediate susceptible to tetracyline, nalidixic acid, ampicillin, cefuroxime sodium and chloramphenicol (Birbir et al., 2016). Although Kluyvera intermedia exhibited positive reactions of $\beta$-galactosidase, ornithine decarboxylase, catalase, nitrate reduction To nitrite, acid production from glucose, arabinose, amygdalin, mannitol, sorbitol and melibiose, negative reaction was detected in citrate utilization, $\mathrm{H}_{2} \mathrm{~S}$ production, oxidase, 
urease, tryptophan deaminase, gelatinase, arginine dihydrolase, lysine decarboxylase, Voges-Proskauer, indol production, acid production from inositol and sucrose (Yazici and Birbir, 2018).

In this study, Kluyvera intermedia was resistant against streptomycin, cephalothin, aztreonam, ampicillin but susceptible against piperacillin-tazobactam, tobramycin, gentamicin, meropenem, cefoxitin, nalidixic acid, ampicillin/sulbactam, tetracyline, norfloxacin, ofloxacin, cefuroxime sodium, sulfamethoxazole-trimethoprim. This isolate was found to be intermediate susceptible to amikacin, kanamycin, chloramphenicol, amoxicillin/clavulanate, ceftriaxone, ciprofloxacin, imipenem and ceftazidime.

In another study, resistance of three Kluyvera intermedia strains, isolated from sink and distinct taps in an intensive care unit in Brazil, against meropenem, imipenem, ertapenem, doripenem was stated by Ribeiro et al. (2014). It was stated that two Kluyvera intermedia strains were susceptible against ceftriaxone, cefepime and aztreonam. In our study, resistance of $K$. intermedia against aztreonam, susceptibility to meropenem and intermediate susceptibility to imipenem and ceftriaxone were detected.

\section{CONCLUSIONS}

This is the first report that investigates antibiotic susceptibility profiles of Ewingella americana and Kluyvera intermedia, isolated from soaked cattle hides and sheep skins, against 24 different antimicrobial agents in leather industry. While E. americana was resistant against three antibiotics (aztreonam, ceftazidime and ceftriaxone), $K$. intermedia was resistant to four antibiotics (streptomycin, cephalothin, aztreonam, ampicillin). Due to resistance of these isolates against the antibiotics used in both human and veterinary medicine, we suggest effective antibacterial applications in leather industry to eradicate these antibiotic resistant microorganisms.

\section{Acknowledgement}

We precisely thank Scientific Research Project Commission of Marmara University for their valuable contribution to our study (FEN-C-YLP-041213-0456).

\section{REFERENCES}

Akpolat, C. et al. (2015), "Molecular Identification of Moderately Halophilic Bacteria and Extremely Halophilic Archaea Isolated From Salted Sheep Skins Containing Red and Yellow Discolorations", JALCA, 110(7), 211-220.

Aslan, E. and Birbir, M. (2012), "Examination of Gram Negative Bacteria on Salt-pack Cured Hides", JALCA, 107(4), 106-115.

Bailey, D.G. and Birbir, M. (1993), "A Study of the Extremely Halophilic Microorganisms Found on Commercially Brine-Cured Cattle Hides”, JALCA, 88(8), 291-299.

Bailey, D.G. and Birbir, M. (1996), "The Impact of Halophilic Organisms on The Grain Quality of Brine Cured Hides", JALCA, 91(2), 47-51.

Bailey, D.G. (2003), "The Preservation of Hides and Skins", JALCA, 98,308-319.

Berber, D. and Birbir, M. (2010), "Examination of Bacterial Populations in Salt, Salted Hides, Soaked Hides and Soak Liquors", JALCA, 105(10), 320-326.

Birbir, M. and Ilgaz, A. (1996), "Isolation and Identification of Bacteria Adversely Affecting Hide and Leather Quality", JSLTC, 80(5), 147-153.

Birbir, M., Ulusoy, K. and Caglayan, P. (2016), "Examination of Multidrug-resistant Enterobacteriaceae Isolated from Salted Cattle Hides and Sheep Skins", JALCA, 111(9), 334-344.

Brenner, D.J. and Farmer, J.J. III. (2005), “Order XIII Enterobacteriales, Family I. Enterobacteriaceae”. in: Brenner, D. J., Krieg, N. R., Staley, J. T and Garrity, G. M.(eds.), Bergey's Manual of Systematic

https://doi.org/10.24264/icams-2018.VI.15 
Bacteriology. The Proteobacteria, The Gammaproteobacteria, 2nd ed., Springer, East Lansing, 587-850, https://doi.org/10.1007/0-387-28022-7.

Bukhari, S.Z. et al. (2008), "Multi-drug Resistant Ewingella americana", Saudi Med J., 29, 1051-1053.

Caglayan, P. et al. (2017), "Screening of Industrially Important Enzymes Produced by Moderately Halophilic Bacteria Isolated from Salted Sheep Skins of Diverse Origin", JALCA, 112(6), 207-216.

CLSI (Clinical and Laboratory Standards Institute) (2014), Performance Standards for Antimicrobial Susceptibility Testing; $24^{\text {th }}$ Informational Supplement, CLSI document M100-S24.

EUCAST (The European Committee on Antimicrobial Susceptibility Testing) (2014), Breakpoint Tables for Interpretation of MICs and Zone Diameters, Version 4.0, www.eucast.org.

European Commission (2013), Tanning of Hides and Skins, Joint Research Centre, European Bureau.

Farmer J.J.III et al. (1985), "Biochemical Identification of New Species and Biogroups of Enterobacteriaceae Isolated from Clinical Specimen”, J Clin Microbiol., 21, 46-76.

Grimont, P.A.D. et al. (1983), "Ewingella americana gen. nov., sp. nov., A New Enterobacteriaceae Isolated from Clinical Specimens", Ann Inst Pasteur Microbiol., 134, 39-52, https://doi.org/10.1016/07692609(83)90102-3.

Hamilton-Miller, J.M.T. and Shah, S. (2001), "Identity and Antibiotic Susceptibility of Enterobacterial Flora of Salad Vegetables”, Int J Antimicrob Agents, 18, 81-83, https://doi.org/10.1016/S0924-8579(01)00353-3.

Heizmann, W.R. and Michel, R. (1991), "Isolation of Ewingella americana froma Patient with Conjunctivitis”, Eur J Clin Microbiol Infect Dis. 10, 957-959, https://doi.org/10.1007/BF02005452.

Janda, J.M. (2006), "New Members of the Family Enterobacteriaceae", in: Dworkin, M., Falkow, S., Rosenberg, E., Schleifer, K. H., Stackebrandt, E. (eds) The Prokaryotes, A Handbook on the Biology of Bacteria, 3rd ed., Volume 6, Proteobacteria:Gamma Subclass, Springer, Minneapolis, 5-40.

Madigan, M.T. and Martinko, J.M. (2015), Brock Biology of Microorganisms, 14th ed., Pearson Education Limited, Edinburg Gate.

Muller, H.E., Fanning, G.R. and Brenner, D.J. (1995), "Isolation of Ewingella americana from Mollusks" Curr Microbiol. 31, 287-290, https://doi.org/10.1007/BF00314581.

Newton, K.G., Harrision, J.C.L and Smith, K.M. (1977), “Coliforms from Hides and Meat.”, Appl Environ Microbiol., 33,199-200.

Oppong, D. et al. (2006), "Application of Molecular Techniques to Identify Bacteria Isolated from the Leather Industry”, JALCA,101, 140-145.

Pavan, M.E. et al. (2005), "Phylogenetic Relationships of the Genus Kluyvera: Transfer of Enterobacter intermedius Izard et al.1980 to the Genus Kluyvera as Kluyvera intermedia comb. nov. and Reclassification of Kluyvera cochleae as a Later Synonym of K. intermedia", Int J Syst Evol Microbiol., 55, 437-442, https://doi.org/10.1099/ijs.0.63071-0.

Pfleiderer, E. and Reiner, R. (1988), "Microorganisms in Processing of Leather" in: Biotechnology Vol $6 b$. Special Microbial Processes H.J.Rehm (ed.), VCH, Weinheim, Germany, 730-739.

Pound, M.W., Tart, S.B. and Okoye, O. (2007), "Multidrug-resistant Ewingella americana: A Case Report and Review of The Literature", Ann Pharmacother., 41, 2066-2070.

Rangarajan, R., Didato, T.D. and Bryant, S. (2003), "Measurement of Bacterial Populations in Typical Tannery Soak Solutions by Traditional and New Approaches", JALCA, 98, 477-485.

Reyes, J.E. et al. (2004), "Prevalence of Ewingella americana in Retail Fresh Cultivated Mushrooms (Agaricus bisporus, Lentinula edodes and Pleurotus ostreatus) in Zaragoza (Spain)", FEMS Microbiol Ecol., 47(3), 291-296, https://doi.org/10.1016/S0168-6496(03)00283-6.

Ribeiro, V.B. et al. (2014), "Detection of blaGES-5 in Carbapenem-Resistant Kluyvera intermedia Isolates Recovered from the Hospital Environment", Antimicrob Agents Chemother., 58(1), 622-623, https://doi.org/10.1128/AAC.02271-13.

Shede, P.N. et al. (2008), "Bacterial Succession on Raw Buffalo Hide and Their Degradative Activities Ambient Storage", Int Biodeterior Biodegradation, 62, 65-74, https://doi.org/10.1016/j.ibiod.2007.12.007.

Stock, I., Sherwood, K.J. and Wiedemann, B. (2003), "Natural Antibiotic Susceptibility of Ewingella americana Strains", J Chemother., 15,428-41.

Ulusoy, K. (2014), "Isolation and Identification of Bacterial Species Belonging to Family Enterobacteriaceae on Salted Hides and Skin Samples and Determination of Their Antibiotic Resistance", Marmara University, Institute of Pure and Applied Sciences.

Ulusoy, K. and Birbir, M. (2015), "Identification and Metabolic Activities of Bacterial Species Belonging to the Enterobacteriaceae on Salted Cattle Hides and Sheep Skins", JALCA, 110(6), 186-199.

World Organization for Animal Health (OIE) (2015), OIE List of Antimicrobial Agents of Veterinary Importance, Geneva: World Health Organization.

Yazici, E. and Birbir, M. (2018), "Examination of Catabolic Activities of Enterobacteriaceae Isolated from Soaked SheepSkins and Cattle Hides", JSLTC, 102(3), 130-136.

***, http://www.bacterio.net/-classifphyla.html\#bacteria, 21.06.2018. 